This article is licensed under the Creative Commons Attribution-NonCommercial 4.0 International License (CC BY-NC) (http://www.karger.com/Services/OpenAccessLicense). Usage and distribution for commercial purposes requires written permission.

\title{
The Journey of an EGFR-Mutant Lung Adenocarcinoma through Erlotinib, Osimertinib and ABCP Immunotherapy Regimens: Sensitivity and Resistance
}

\author{
Albina Kibirova ${ }^{a}$ Malcolm D. Mattes ${ }^{b-d}$ Matthew Smolkin ${ }^{c, e}$ \\ Patrick C. $\mathrm{Ma}^{f}$ \\ aSection of Hematology/Oncology, Department of Medicine, West Virginia University \\ School of Medicine, Morgantown, WV, USA; bepartment of Radiation Oncology, WVU \\ Cancer Institute, West Virginia University, Morgantown, WV, USA; ${ }^{\circ} W V U$ Cancer Institute, \\ West Virginia University, Morgantown, WV, USA; ${ }^{d}$ West Virginia Clinical and Translational \\ Science Institute, Morgantown, WV, USA; eSection of Molecular Pathology, Department of \\ Pathology, West Virginia University School of Medicine, Morgantown, WV, USA; ${ }^{\mathrm{P}}$ Penn \\ State Cancer Institute, Penn State Health Milton S. Hershey Medical Center, Penn State \\ University, Hershey, PA, USA
}

\section{Keywords}

Anti-angiogenesis · Drug resistance · EGFR · Immunotherapy · Non-small cell lung cancer

\begin{abstract}
Patients with epidermal growth factor receptor (EGFR) mutation positive non-small cell lung cancer (NSCLC) have several EGFR targeting tyrosine kinase inhibitors (TKIs) available in frontline management. However, the disease will inevitably progress over time due to acquired resistance. Longitudinal tumor profiling for genomics guided therapy is indicated upon disease
\end{abstract}


progression. It is a common scenario yet, when after failure of EGFR-TKIs, potentially actionable genomic alterations are lacking. Management of such patient is challenging with very limited options available. Combination of chemotherapy, anti-vascular/anti-angiogenic and immunecheckpoint inhibitors may become a salvage option for such patients. Here we describe a case of TKI refractory EGFR-mutant NSCLC successfully treated with carboplatin, paclitaxel, atezolizumab and bevacizumab combination with remarkable prompt tumor response.

(C) 2019 The Author(s)

Published by S. Karger AG, Basel

\section{Introduction}

Despite excellent initial overall response to targeted EGFR (see Table 1 for glossary) therapy reaching 70-80\%, essentially all patients with EGFR-mutant NSCLC inevitably progress over time due to acquired drug resistance, thereby limiting their long term survival benefits. Multiple mechanisms of acquired resistance against first-generation EGFR TKIs (erlotinib and gefitinib) have been described, with about half of the cases attributed to the exon 20 gatekeeper EGFR-T790M (c.2369C>T) mutation [1-3].

Osimertinib is the only available FDA-approved TKI active against T790M, and recently it has further been approved as first-line therapy in EGFR mutant lung cancer patients based on the FLAURA study [4]. In this study, the median duration of response to osimertinib reached 17.6 months ( $95 \%$ CI, 13.8 to 22.0 ) as compared to 8.5 months (95\% CI, 7.3 to 9.8) with first generation TKIs [4]. For patients who develop disease progression on EGFR TKIs, further treatment options are limited. This is particularly true for those who progress on osimertinib, with emerging drug-resistant C797S $(c .2390 G>C)$ mutation, which currently is lacking standard therapeutic options [5].

Cytotoxic chemotherapy only yields limited and short-lived responses in general with numerous adverse effects. Attempts to incorporate immune checkpoint inhibitors in patients with EGFR-driven NSCLC have initially been also disappointing, especially when used as a single agent programmed death-1 (PD-1) or PD-ligand 1 (PD-L1) antibody [6-8]. Recently, promising data has emerged with overcoming immune checkpoint inhibition resistance by combining it with anti-angiogenic agents. It is believed that anti-vascular endothelial growth factor (VEGF) not only promotes anti-angiogenesis but also acts as a key mediator of the immunosuppressive microenvironment that enables tumor cells to evade immune surveillance [9]. Combining anti-VEGF and immune checkpoint inhibitors has recently been shown to work synergistically in several tumor types.

\section{Case Report}

A 45-years-old Caucasian female, who was a never-smoker and previously healthy, presented with progressive forgetfulness and confusion. MRI of the brain demonstrated multiple metastatic lesions, with the dominant mass in the left frontal lobe measuring $1.5 \mathrm{~cm}$ associating with extensive vasogenic edema. She was diagnosed with metastatic lung adenocarcinoma of right upper lung with involvement of mediastinal lymph nodes, brain, liver and bones. She 
Kibirova et al.: The Journey of an EGFR-Mutant Lung Adenocarcinoma through Erlotinib, Osimertinib and ABCP Immunotherapy Regimens: Sensitivity and Resistance

underwent craniotomy with resection of the largest brain lesion revealing the diagnosis. Histological and immunohistochemical tumor characteristics are illustrated in Fig. 1. Patient then received stereotactic radiosurgery to the 8 largest brain metastatic lesions followed by whole brain radiotherapy. As part of a comprehensive molecular tumor profiling (Caris Molecular Intelligence; Caris Life Sciences, Phoenix, AZ), her tumor was found to be positive for EGFR exon 19 deletion (E746-A750del) and a TP53 mutation (Table 2). It was negative for $A L K$ or ROS1 rearrangements, KRAS or BRAF mutations and also negative for PD-L1 expression.

Patient was started on erlotinib $150 \mathrm{mg}$ daily as first-line therapy and had a near-complete response on follow-up restaging imaging. Nine months later, she developed oligo-progressive disease with two new skeletal lesions which were treated with local stereotactic body radiotherapy and erlotinib was continued at that time. Over the following 8 months, a similar approach was applied to several other gradually progressive skeletal metastases while further therapeutic options were explored. One of these drug-resistant metastatic skeletal lesion in the pelvis was rebiopsied but yielded no informative molecular insights on repeat molecular profiling. Circulating tumor DNA (ctDNA; also referred to as cell-free DNA, cfDNA) liquid biopsy testing (Guardant360; Guardant Health; Redwood City, CA) was performed and detected the presence of T790M mutation (5.4\% of altered cell-free DNA (Fig. 2, Fig. 3A). Based on this finding, patient was started on osimertinib $80 \mathrm{mg}$ daily. She again had an excellent response with a progression-free duration lasting for 12 months. Subsequently she again developed oligoprogressive skeletal metastases, which were successfully managed with local radiotherapy concurrently with osimertinib over the next 6 months. She ultimately progressed on osimertinib more extensively beyond further radiotherapy control on TKI, including multiple mediastinal nodal progression. Mediastinal lymph node rebiopsy was obtained confirming adenocarcinoma histology without transformation, but material was insufficient for broad-based molecular testing. Repeat cfDNA liquid biopsy revealed no EGFR mutations (Fig. 3B).

For the third-line of treatment patient was started on a quadruplet combination of carboplatin AUC 6, paclitaxel $200 \mathrm{mg} / \mathrm{m}^{2}$, bevacizumab $15 \mathrm{mg} / \mathrm{kg}$ and atezolizumab 1,200 $\mathrm{mg}$ (ABCP), based on encouraging data from the IMpower 150 study [10]. The first treatment cycle was complicated by subclinical thyroiditis, grade 3 nausea, vomiting and pancytopenia requiring hospital admission. The second cycle was delayed with also a dose reduction on the cytotoxics. Nevertheless, restaging PET/CT scan at week 6 after only one cycle of treatment already demonstrated a near-complete response (Fig. 4). Patient subsequently completed total of 4 cycles of ABCP followed by maintenance bevacizumab and atezolizumab (AB). She remained in radiographic remission for 9.5 months when her repeat restaging PET/CT scan demonstrated enlarging FDG-avid primary RUL lung nodule and several new skeletal lesions and brain MRI revealed new tiny enhancing foci in right frontal and left parietal cerebral cortex. At this time, patient was agreeable for treatment with repeat local radiotherapy to drugresistant disease lesions while continuing immune checkpoint PD-L1 therapy on atezolizumab maintenance. Bevacizumab was temporarily held during the time of radiotherapy. She has received GKRS to brain lesions and the plan is to continue with focal radiation to skeletal metastases. Repeat cfDNA liquid biopsy profiling at time of $\mathrm{ABCP} / \mathrm{AB}$ regimen acquired resistant progression revealed re-emergence of EGFR exon 19 deletion and new emergence of $C D K 6$ amplification and ATM R3008C mutation (Fig. 3C). Besides, there was a new CDK12 mutation of unknown significance; and the TP53 as well as EGFR T790M mutations remained undetectable. Overall, it was determined that no new readily targetable alterations were found. 


\section{Case Reports in Oncology}

Case Rep Oncol 2019;12:765-776

DOI: $10.1159 / 000503417$

(c) 2019 The Author(s). Published by S. Karger AG, Basel www.karger.com/cro

Kibirova et al.: The Journey of an EGFR-Mutant Lung Adenocarcinoma through Erlotinib Osimertinib and ABCP Immunotherapy Regimens: Sensitivity and Resistance

\section{Discussion/Conclusion}

Despite the advent of targeted EGFR-TKIs like erlotinib and osimertinib, the development of drug resistance remains a formidable challenge in the management of EGFR-mutant NSCLC. Multiple mechanisms of acquired drug resistance have been described in the literature, supporting longitudinal tumor profiling for genomics guided therapy. While T790M mutation represents the dominant mutational resistance mechanism against early generation EGFR-TKIs, there is emerging data describing an acquired mutation in C797 codon of EGFR leading to osimertinib resistance [5]. This mutation has no therapeutic options presently although there is potential 4th generation EGFR-TKI in clinical development [11]. Other possible mechanisms of resistance include activation of alternative bypass pathways (MET, HER2, KRAS, BRAF, PIK3CA) or oncogenic fusions (CCDC6-RET, FGFR3-TACC3, EML4-ALK), some of which can be exploited with therapeutic interventions $[1,2,12]$.

In a real life practice however, as in our presented case, by the time acquired EGFR-TKI resistance develops, commercially available effective therapeutic options are limited and clinical trials are not always readily available. The recent exciting achievement of targeting the immune checkpoint pathway in cancer therapy including NSCLC has not yet significantly impacted patients with most oncogenic-driver mutations, especially in EGFR and ALK. Most, if not all, of the clinical studies testing immune checkpoint inhibitors excluded patients with known $E G F R$ mutations and $A L K$-rearrangements. Studies in second-line immunotherapy trials in EGFR-mutated NSCLC patients yielded disappointing response and failed to demonstrate any survival advantage when compared with docetaxel [6-8]. Moreover, combination strategy with addition of immunotherapy to targeted TKIs have also proven to be associated with significant treatment toxicities [13]. Oncogene-driven lung tumors, e.g. EGFR mutation and $A L K$-rearrangement, were found to correlate with low tumor mutational burden and often also low PD-L1 expression [14]. It is conceivable that these factors above could account for the relative lack of response and treatment efficacy of immune checkpoint inhibitors in this patient population. Furthermore, even in those with high PD-L1 expression, this was not highly predictive for positive responses either.

The anti-VEGF antibody bevacizumab combined with chemotherapy was approved for the treatment of metastatic NSCLC in combination with carboplatin and paclitaxel based on the ECOG 4599 study in 2006 [16]. The anti-VEGF strategy has been investigated in the IMpower 150 study as a potentiating option in combination with the immune checkpoint PD-L1 inhibitor atezolizumab [10]. The concept behind combining anti-angiogenic agents with immune checkpoint inhibitors is at least partially based on the demonstration that VEGF is not only important for tumor neovascularization, but is also a key immuno-modulating factor produced by solid tumors to evade immune recognition and tumor cell killing $[9,15]$. The IMpower 150 study included a small cohort of EGFR mutation positive NSCLC patients (35/400 or $8.8 \%$ ) who progressed on prior EGFR-TKI therapy and were assigned to receive ABCP regimen in comparison with patients who received the same regimen without atezolizumab (BCP). In the subgroup analysis, the median progression-free survival (PFS) in patients with EGFR mutation or $A L K$-rearrangement in the ABCP combination arm was 9.7 months which was significantly longer compared to the BCP arm, 6.1 months respectively with unstratified hazard ratio 0.59 . The difference was favorably comparable to the entire study cohort of NSCLC patients receiving ABCP combination [10]. 


\section{Case Reports in Oncology}

Case Rep Oncol 2019;12:765-776

DOI: $10.1159 / 000503417$

2019 The Author(s). Published by S. Karger AG, Basel www.karger.com/cro

Kibirova et al:: The Journey of an EGFR-Mutant Lung Adenocarcinoma through Erlotinib, Osimertinib and ABCP Immunotherapy Regimens: Sensitivity and Resistance

The presented case herein illustrates a classic natural history of EGFR-mutant NSCLC. It also provides support for the utility of consolidative local radiotherapy against oligo-progressive lesions beyond EGFR TKI acquired drug resistance. After repeated resistance to erlotinib and osimertinib, our patient ultimately achieved a near-complete response after only one cycle of $A B C P$ regimen, demonstrating the possible prompt and remarkable efficacy of the combination strategy in this heavily-pretreated disease scenario. Our patient continued to do well on maintenance $\mathrm{AB}$ treatment for 9.5 months of progression-free interval with excellent performance status, and overall $>4$ years since her initial metastatic cancer diagnosis. Notably, her original tumor stained negative for PD-L1 expression and highlighted rare CD4 and CD8 positive lymphocytes (Fig. 1). However, the 9.5 months duration of response was less than ideal but was also comparable with the outcome in the original IMpower 150 study. She then developed disease progression including CNS involvement. Repeat cfDNA liquid biopsy on $\mathrm{ABCP} / \mathrm{AB}$ progression demonstrated no readily targetable alterations. Our knowledge on molecular mechanisms in $\mathrm{ABCP}$ acquired resistant diseases is very limited at present, but expectedly more studies would continue to emerge in the near future. To our best knowledge, this is the first molecular profiling report of a case of $A B C P$ regimen acquired drug resistant progression. Interestingly, our liquid biopsy molecular profiling at $\mathrm{ABCP} / \mathrm{AB}$ acquired resistant progression identified novel molecular aberrations, i.e. CDK6 amplification, ATM R3008C, that might represent the genomic driving events behind the drug resistance development on combination of cytotoxic chemotherapy with anti-angiogenic and immune checkpoint inhibitors. While CDK6 amplification is associated with CDK inhibitor resistance thus negating such therapeutic option for our patient, the ATM mutation leading to genomic instability might offer a novel therapeutic opportunity with a PARP and/or an ATM/ATR inhibitor [17].

In conclusion, the PD-L1 immune checkpoint therapy incorporated ABCP regimen provides a promising salvage therapeutic option for patients with EGFR-mutation driven NSCLC resistant to targeted TKIs, especially beyond osimertinib. The data from IMpower 150 study provides further support to the development of combinational strategies using chemotherapy, anti-vascular/anti-angiogenic and immune checkpoint inhibitors in these patients. However, clinically validated predictive biomarkers for response to immunotherapy-containing salvage regimens for these patients are still grossly lacking. It is also unclear at present time how the $\mathrm{ABCP}$ regimen will compare to secondary targeted therapy approaches if they are uncovered on repeat biopsy and genomic profiling. We propose that randomized clinical studies using this novel combinational strategy should be conducted with a focus on EGFR-mutant and other oncogene-addicted NSCLC in order to fully validate this clinical hypothesis and to resolve the related clinical issues outlined above.

\section{Statement of Ethics}

This report and related work were conducted in compliance with the guidelines for human studies and Institution Review Board approved study protocol. It is also affirmed that the subject has given her written informed consent to publish her case (including publication of images). 


\section{Disclosure Statement}

Patrick C. Ma: Speaker Bureau: Takeda, AstraZeneca, Merck, Bristol-Myers Squibb, Bayer. Advisory Board: AstraZeneca, Apollomics, Takeda. Research Funding (to institution): AstraZeneca, Bristol-Myers Squibb, Apollomics, Epicentrix, Merck, Pfizer, Loxo/Bayer.

Albina Kibirova, Malcolm D. Mattes and Matthew Smolkin have no financial relationships to disclose.

\section{Funding Sources}

This work was funded by the WVU Cancer Institute, West Virginia University and Penn State Cancer Institute, Penn State Health Milton S. Hershey Medical Center.

\section{Author Contributions}

Albina Kibirova and Patrick C Ma conceived the study concept and design. All authors participated in the clinical care of the subject, manuscript writing and revision. All authors approved the final manuscript to be submitted.

\section{References}

1 Westover D, Zugazagoitia J, Cho BC, Lovly CM, Paz-Ares L. Mechanisms of acquired resistance to first- and second-generation EGFR tyrosine kinase inhibitors. Ann Oncol. 2018 Jan;29 suppl_1:i10-9.

2 Morgillo F, Della Corte CM, Fasano M, Ciardiello F. Mechanisms of resistance to EGFR-targeted drugs: lung cancer. ESMO Open. 2016 May;1(3):e000060.

3 Mok TS, Wu YL, Ahn MJ, Garassino MC, Kim HR, Ramalingam SS, et al.; AURA3 Investigators. Osimertinib or platinum-pemetrexed in EGFR T790M-positive lung cancer. N Engl J Med. 2017 Feb;376(7):629-40.

4 Soria JC, Ohe Y, Vansteenkiste J, Reungwetwattana T, Chewaskulyong B, Lee KH, et al.; FLAURA Investigators. Osimertinib in untreated EGFR-mutated advanced non-small-cell lung cancer. N Engl J Med. 2018 Jan;378(2):113-25.

5 Oxnard GR, Hu Y, Mileham KF, Husain H, Costa DB, Tracy P, et al. Assessment of resistance mechanisms and clinical implications in patients with EGFR T790M-positive lung cancer and acquired resistance to osimertinib. JAMA Oncol. 2018 Nov;4(11):1527-34.

6 Borghaei H, Paz-Ares L, Horn L, Spigel DR, Steins M, Ready NE, et al. Nivolumab versus docetaxel in advanced nonsquamous non-small-cell lung cancer. N Engl J Med. 2015 Oct;373(17):1627-39.

7 Lee CK, Man J, Lord S, Cooper W, Links M, Gebski V, et al. Clinical and molecular characteristics associated with survival among patients treated with checkpoint inhibitors for advanced non-small cell lung carcinoma: A systematic review and meta-analysis. JAMA Oncol. 2018 Feb;4(2):210-6.

8 Lee CK, Man J, Lord S, Links M, Gebski V, Mok T, et al. Checkpoint inhibitors in metastatic EGFR-mutated non-small cell lung cancer-A meta-analysis. J Thorac Oncol. 2017 Feb;12(2):403-7.

9 Manegold C, Dingemans AC, Gray JE, Nakagawa K, Nicolson M, Peters S, et al. The Potential of combined immunotherapy and antiangiogenesis for the synergistic treatment of advanced NSCLC. J Thorac Oncol. 2017 Feb;12(2):194-207.

10 Socinski MA, Jotte RM, Cappuzzo F, Orlandi F, Stroyakovskiy D, Nogami N, et al.; IMpower150 Study Group. Atezolizumab for first-line treatment of metastatic nonsquamous NSCLC. N Engl J Med. 2018 Jun;378(24):2288-301.

11 Wang S, Song Y, Liu D. EAI045: the fourth-generation EGFR inhibitor overcoming T790M and C797S resistance. Cancer Lett. 2017 Jan;385:51-4. 


\section{Case Reports in Oncology}

Case Rep Oncol 2019;12:765-776

DOI: $10.1159 / 000503417$

(C) 2019 The Author(s). Published by S. Karger AG, Basel www.karger.com/cro

Kibirova et al: The Journey of an EGFR-Mutant Lung Adenocarcinoma through Erlotinib, Osimertinib and ABCP Immunotherapy Regimens: Sensitivity and Resistance

12 Ou SI, Horn L, Cruz M, Vafai D, Lovly CM, Spradlin A, et al. Emergence of FGFR3-TACC3 fusions as a potential by-pass resistance mechanism to EGFR tyrosine kinase inhibitors in EGFR mutated NSCLC patients. Lung Cancer. 2017 Sep;111:61-4.

13 Ahn MJ, Sun JM, Lee SH, Ahn JS, Park K. EGFR TKI combination with immunotherapy in non-small cell lung cancer. Expert Opin Drug Saf. 2017 Apr;16(4):465-9.

14 Dong ZY, Zhang JT, Liu SY, Su J, Zhang C, Xie Z, et al. EGFR mutation correlates with uninflamed phenotype and weak immunogenicity, causing impaired response to PD-1 blockade in non-small cell lung cancer. OncoImmunology. 2017 Jul;6(11):e1356145.

15 Ohm JE, Carbone DP. VEGF as a mediator of tumor-associated immunodeficiency. Immunol Res. 2001;23(23):263-72.

16 Sandler A, Gray R, Perry MC, Brahmer J, Schiller JH, Dowlati A, et al. Paclitaxel-carboplatin alone or with bevacizumab for non-small-cell lung cancer. N Engl J Med. 2006 Dec;355(24):2542-50.

17 Perkhofer L, Schmitt A, Romero Carrasco MC, Ihle M, Hampp S, Ruess DA, et al. ATM deficiency generating genomic instability sensitizes pancreatic ductal adenocarcinoma cells to therapy-induced DNA damage. Cancer Res. 2017 Oct;77(20):5576-90.

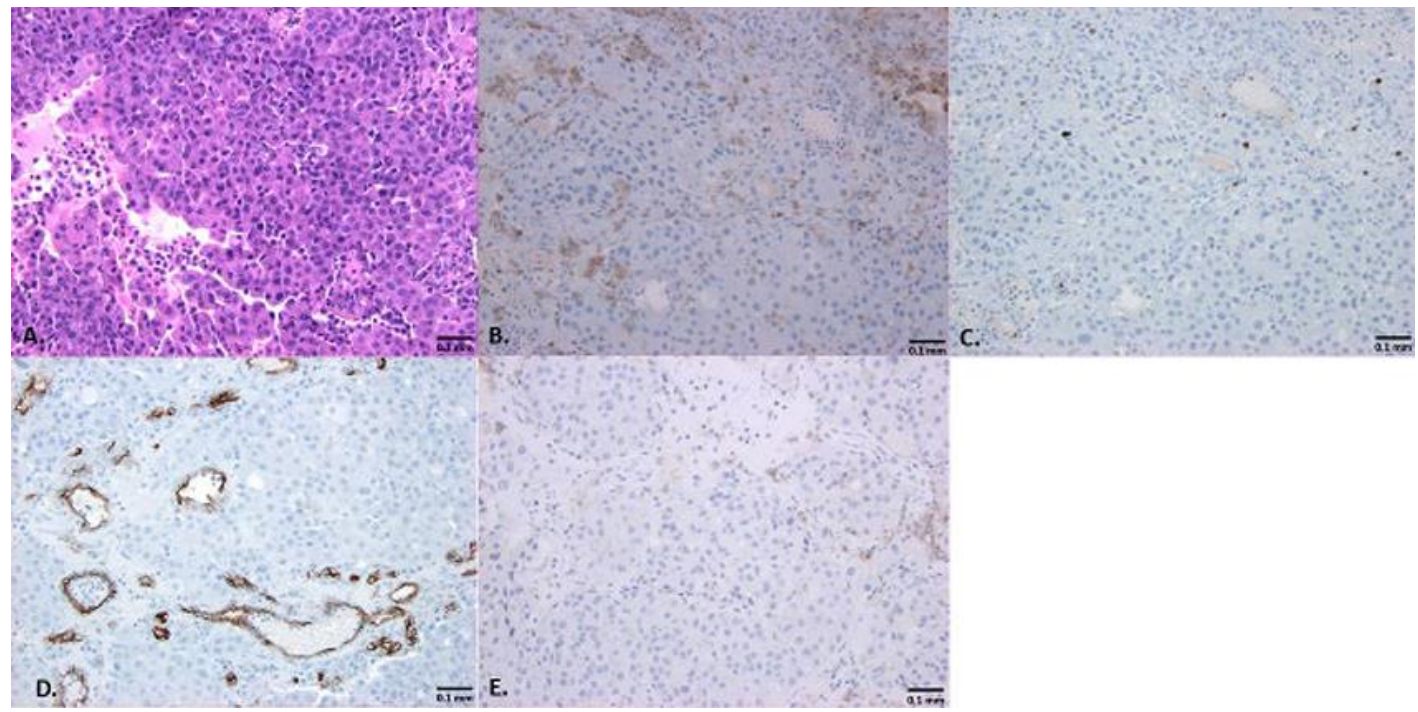

Fig. 1. Histological and immunohistochemical characteristics of the metastatic tumor. A. Metastatic adenocarcinoma to the brain (H\&E at 200x); B. An immunohistochemical stain for CD4 at 200× highlights rare lymphocytes and non-specific staining; C. An immunohistochemical stain for CD8 at 200× highlights rare lymphocytes; D. A CD34 immunohistochemical stain at 200× highlights endothelium; E. An immunohistochemical stain for PD-L1 (22C3) highlights rare weak tumor cell membranous staining, rare positive staining in lymphocytes. 


\section{Case Reports in Oncology}

Kibirova et al.: The Journey of an EGFR-Mutant Lung Adenocarcinoma through Erlotinib

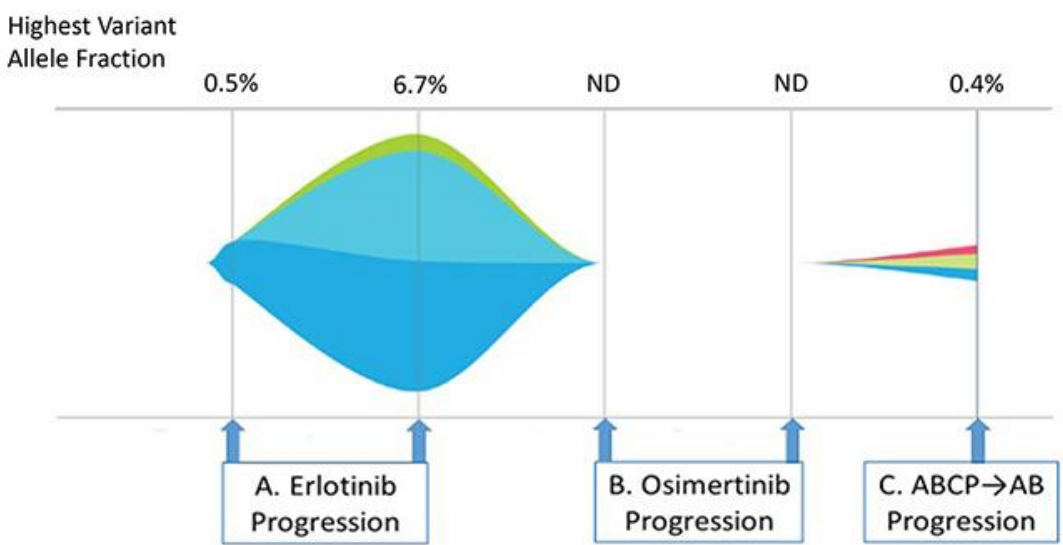

Fig. 2. Summary longitudinal liquid cfDNA profiling (Guardant360) with the tumor response map. 
A.

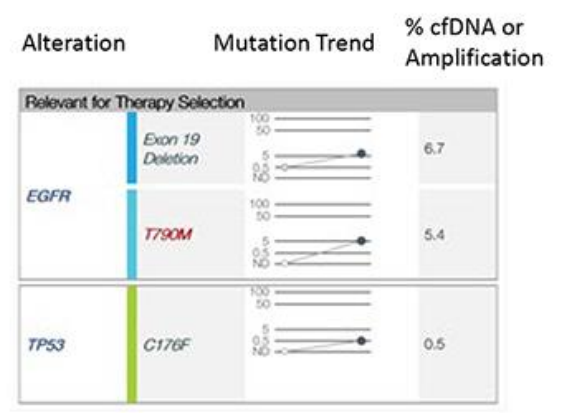

B.

(c) 2019 The Author(s). Published by S. Karger AG, Basel www.karger.com/cro

Kibirova et al: The Journey of an EGFR-Mutant Lung Adenocarcinoma through Erlotinib, Osimertinib and ABCP Immunotherapy Regimens: Sensitivity and Resistance

C.

\begin{tabular}{|c|c|c|c|}
\hline Alteration & $\%$ cfDNA or Amp & Alteration/Mutation T & \\
\hline CDK12N1208S & $0.4 \%$ & o. & $\begin{array}{l}\text { Variant of Uncertain } \\
\text { Significances }\end{array}$ \\
\hline $\begin{array}{l}\text { EGFRE746_A750del } \\
\text { (Exon } 19 \text { deletion) }\end{array}$ & $0.3 \%$ & osm arm No & \\
\hline ATM R $3008 \mathrm{C}$ & $0.2 \%$ & 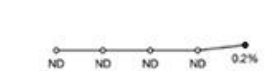 & \\
\hline $\begin{array}{l}\text { CDK6 Amplification } \\
\text { Amplifications not graphed above }\end{array}$ & Medium (t+) & so no vof & \\
\hline TP53C176F & ND & No asm in :0 & \\
\hline EGFRT79OM & ND & No 54 a no 100 & \\
\hline
\end{tabular}

Fig. 3. Longitudinal liquid cfDNA profiling (Guardant360) results. (A) New emergence of acquired EGFR T790M mutation with 5.4\% allele frequency of altered circulating cell-free DNA (\% cfDNA) demonstrated on erlotionib progression, which disappeared in the following 2 serial liquid biopsies while on osimertinib, during profiling upon drug resistance to osimertinib (B). Subsequent profiling on ABCP progression revealed the presence of initial driver EGFR exon 19 deletion (E746-A750del specific) without EGFR T790M mutation, and new additional alterations (CDK12 N1208S, ATM R3008C and CDK6 amplification) (C). 


\section{Case Reports in Oncology}
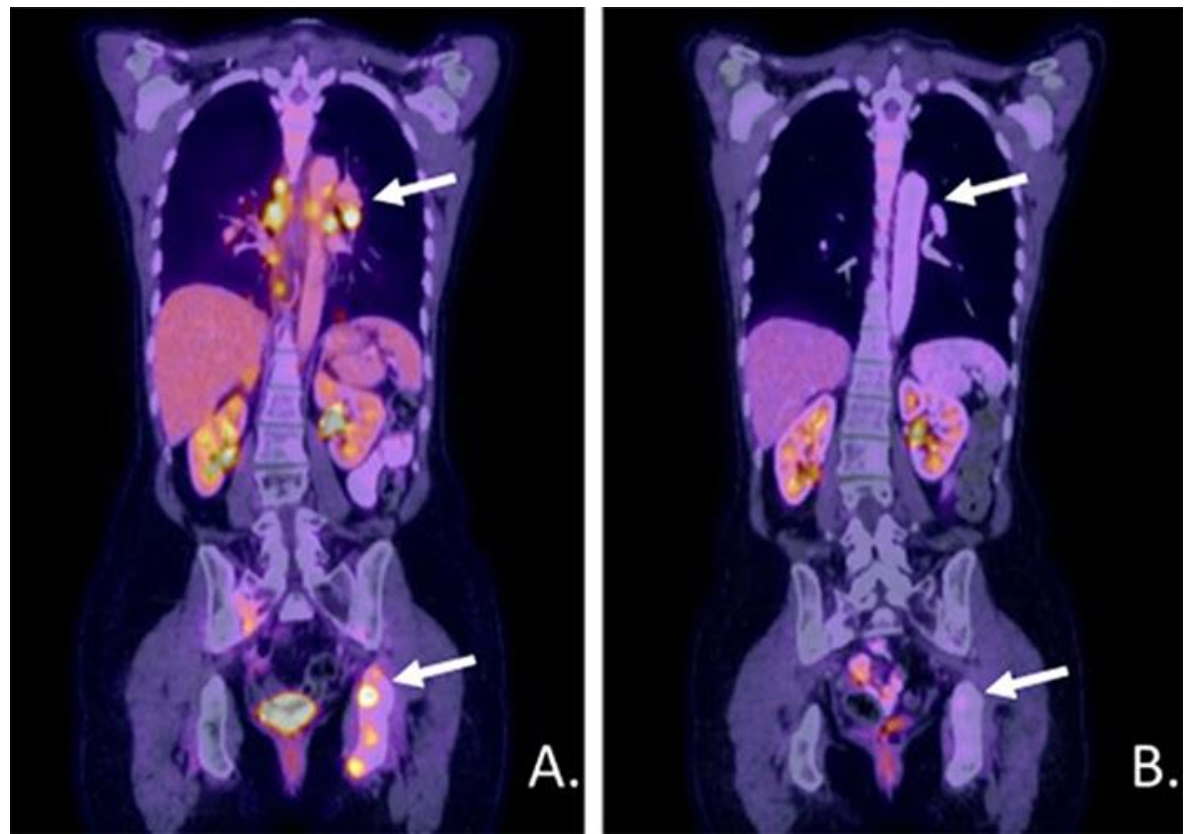

Fig. 4. PET/CT scans prior to initiation of ABCP therapy (A) and after one cycle of treatment (B), shown. Remarkable and prompt near-complete response with radiographic and metabolic resolution of extensive mediastinal lymphadenopathy and left pelvis bony metastases in resistant progression against osimertinib was noted after the 1st cycle of ABCP salvage treatment (arrows). 
Table 1. Glossary of genomic terms and nomenclature

\begin{tabular}{|c|c|}
\hline EGFR & Epidermal growth factor receptor, transmembrane receptor tyrosine kinase \\
\hline $\begin{array}{l}\text { T790M } \\
(\text { c. } 2369 \mathrm{C}>\mathrm{T})\end{array}$ & $\begin{array}{l}\text { This pathogenic mutation occurs within exon } 20 \text { of EGFR kinase domain and results } \\
\text { in an amino acid substitution at position } 790 \text { from a threonine (T) to a methionine } \\
\text { (M) }\end{array}$ \\
\hline $\begin{array}{l}\text { C797S } \\
(\text { c. } 2390 G>C)\end{array}$ & $\begin{array}{l}\text { This pathogenic mutation occurs within exon } 20 \text { of EGFR kinase domain and results } \\
\text { in an amino acid substitution at position } 797 \text { in EGFR, from a cysteine (C) to a serine } \\
\text { (S) }\end{array}$ \\
\hline PD-1 & $\begin{array}{l}\text { Programmed cell death protein } 1 \text {, is a receptor expressed on T-cells, activation of } \\
\text { which causes suppression of immune response }\end{array}$ \\
\hline PD-L1 & $\begin{array}{l}\text { Programmed death ligand } 1 \text {, transmembrane protein, regulating immune response } \\
\text { by interacting with PD-1 receptor }\end{array}$ \\
\hline $\begin{array}{l}\text { Exon } 19 \text { deletion } \\
\text { (E746-A750del) }\end{array}$ & $\begin{array}{l}\text { In-frame deletions occurring within exon } 19 \text { of the EGFR kinase domain, one of the } \\
\text { most common EGFR mutations in NSCLC }\end{array}$ \\
\hline TP53 & Tumor protein p53 is a gene that codes for a tumor suppressor protein \\
\hline ALK & Anaplastic lymphoma kinase, transmembrane receptor tyrosine kinase \\
\hline RoS1 & ROS proto-oncogene 1 , integral membrane protein receptor tyrosine kinase \\
\hline KRAS & KRAS proto-oncogene, GTPase \\
\hline BRAF & B-Raf proto-oncogene, serine/threonine kinase \\
\hline TPS & Tumor proportion score \\
\hline MET & c-Met protooncogene \\
\hline FISH & Fluorescence In Situ Hybridization \\
\hline CDK12 & $\begin{array}{l}\text { Cyclin dependent arginine-serine kinase } 12 \text {, regulating cell cycle progression and } \\
\text { transcription }\end{array}$ \\
\hline ATM & $\begin{array}{l}\text { Ataxia-Telangiectasia serine/threonine kinase, involved in double-strand DNA dam- } \\
\text { age repair }\end{array}$ \\
\hline CDK6 & $\begin{array}{l}\text { Cyclin dependent serine/threonine protein kinase } 6 \text {, regulating cell cycle progression } \\
\text { and transcription }\end{array}$ \\
\hline ATR & $\begin{array}{l}\text { Ataxia telangiectasia and Rad3-related serine/threonine-protein kinase, involved in } \\
\text { single-strand DNA damage repair }\end{array}$ \\
\hline
\end{tabular}

Kibirova et al.: The Journey of an EGFR-Mutant Lung Adenocarcinoma through Erlotinib,

Osimertinib and ABCP Immunotherapy Regimens: Sensitivity and Resistance

(C) 2019 The Author(s). Published by S. Karger AG, Basel www.karger.com/cro 
Table 2. Comprehensive tumor molecular profiling analysis of case tumor specimen by Caris Molecular Intelligence (CMI)

\section{Genes tested with alterations}

\begin{tabular}{lllll}
\hline Gene & Alteration & Frequency, \% & Exon & Result \\
\hline EGFR & E746_A750del & 71 & 19 & Mutated, pathogenic \\
TP53 & C176F & 42 & 5 & Mutated, pathogenic \\
\hline
\end{tabular}

Genes tested with no mutations detected

\begin{tabular}{llllll}
\hline ABL1 & BRCA2 & Her2/neu (ERBB2) & IDH1 & NOTCH1 & ROS1 \\
AKT1 & c-KIT & ERBB3 & IDH2 & NRAS & SMO \\
ALK & CDK4 & FGFR1 & JAK2 & NTRK1 & SRC \\
Androgen receptor & CDKN2A & FGFR2 & KDR (VEGFR2) & PDGFRA & VHL \\
APC & CHEK1 & FGFR3 & KRAS & PDGFRB & WT1 \\
ARAF & CHEK2 & FLT3 & MEK1 & PIK3CA \\
ATM & c-MET & GNA11 & MEK2 & PTCH1 \\
BAP1 & CSF1R & GNAQ & MLH1 & PTEN \\
BRAF & CTNNB1 & GNAS & MPL & RAF1 \\
BRCA1 & DDR2 & HRAS & NF1 & RET \\
\hline
\end{tabular}

Kibirova et al.: The Journey of an EGFR-Mutant Lung Adenocarcinoma through Erlotinib Osimertinib and ABCP Immunotherapy Regimens: Sensitivity and Resistance 